\title{
Comparison of BCF-10, BCF-12, and BCF-20 Scintillating Fibers for Use in a 1-Dimensional Linear Sensor
}

\section{IEEE 2012 Nuclear Science Symposium}

David L. Chichester

Scott M. Watson

James T. Johnson

The INL is a

U.S. Department of Energy

National Laboratory

operated by

Battelle Energy Alliance

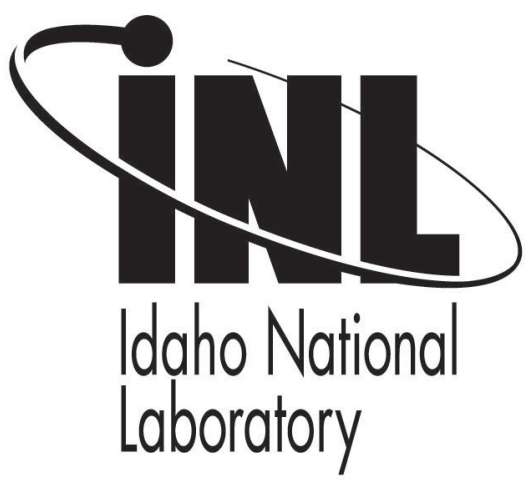

This is a preprint of a paper intended for publication in a journal or proceedings. Since changes may be made before publication, this preprint should not be cited or reproduced without permission of the author. This document was prepared as an account of work sponsored by an agency of the United States Government. Neither the United States Government nor any agency thereof, or any of their employees, makes any warranty, expressed or implied, or assumes any legal liability or responsibility for any third party's use, or the results of such use, of any information, apparatus, product or process disclosed in this report, or represents that its use by such third party would not infringe privately owned rights. The views expressed in this paper are not necessarily those of the United States Government or the sponsoring agency. 


\title{
Comparison of BCF-10, BCF-12, and BCF-20 Scintillating Fibers for Use in a 1-Dimensional Linear Sensor
}

\author{
David L. Chichester, Senior Member, IEEE, Scott M. Watson, and James T. Johnson
}

\begin{abstract}
One-dimensional fiber-bundle arrays may prove useful in a number of radiation sensing applications where radiation detection over large areas is needed. Tests have been performed to evaluate the light generation and transmission characteristics of 15-meter long, 10-fiber bundles of BCF-10, BCF-12, and BCF-20 scintillating fibers (Saint Gobain) exposed to collimated gamma-ray sources. The test set-up used one R9800 (Hamamatsu) photomultiplier tube (PMT) at each end, with a high-speed waveform digitizer to collect data. Time constraints were imposed on the waveform data to perform timeof-flight analysis of the events in the fiber bundles, eliminating spurious noise pulses in the high gain PMTs and also allowing 1dimensional localization of interactions along the lengths of the fiber bundles. This paper will present the results of these measurements including the attenuation coefficients of the three fiber types and the timing resolution (position uncertainty) possible for each fiber bundle when using the R9800 PMTs.
\end{abstract}

\section{INTRODUCTION}

$\mathrm{L}^{\mathrm{s}}$ ong, small-diameter scintillating fiber bundle (SFB) $\sim_{\text {assemblies are under investigation at Idaho National }}$ Laboratory (INL) with the goal of developing onedimensional (1-D) linear sensors that may be used for monitoring and characterizing ionizing radiation fields over long distances or over large areas. There are several needs for low-technology, distributed sensor networks that are capable of monitoring radiation fields including radiation health physics, system monitoring at large facilities (nuclear reactors, nuclear material processing facilities, high-energy particle accelerators, hospitals, etc.), and nuclear security and nonproliferation applications. Traditional approaches involve the emplacement of discrete instrumentation at closely-spaced intervals; continuous sensors have the ability to eliminate gaps or weak-spots in between such sensors modules, while at the same time reducing support infrastructure requirements including installed equipment mounting and power consumption. This paper presents an overview of prior research in this area and then presents the results of recent

Manuscript received November 11, 2012. Idaho National Laboratory is operated for the U.S. Department of Energy by Battelle Energy Alliance under DOE contract DE-AC07-05-ID14517.

D. L. Chichester (telephone: 208-526-8920, e-mail: david.chichester@inl.gov), S. M. Watson (telephone: 208-525-0572, e-mail: scott.watson@inl.gov) and J. T. Johnson (telephone: 208-525-0572, email:james.johnson@inl.gov) are all with Idaho National Laboratory, Idaho Falls, Id., 83415, USA.

The author's would like to acknowledge the support of Idaho National Laboratory's Laboratory-Directed Research and Development (LDRD) program for this work. studies performed to assess the performance of three different candidate SFB materials.

\section{BACKGROUND INFORMATION}

Early work using SFB assemblies for radiation detection and measurement primarily dealt with using thousands of short length $(\sim 10-\mathrm{cm}$ long) scintillating fibers, such as NE103 , bundled together to create a single sensor unit, usually in the form of a right cylinder using plastic fibers embedded in a plastic matrix.[1,2] This form factor, originally developed for space research, was found useful as a tool for providing a directionally sensitive neutron probe and was later expanded and improved upon for terrestrial survey applications. Later, other researchers continued these efforts and subsequently developed several other scintillating fiber detectors for measuring neutrons in the same cylindrical form factor.[3-8] Overtime detector developers eventually began to use boronor lithium-based fibers in some cases to increase the thermal neutron sensitivity of their instruments.

As the technology needed to manufacture longer length fiber optics was developed, interest in using longer length scintillating fibers as radiation instrumentation grew as well, along two different paths. In one case researchers chose to use newly available long-length fibers to create neutron and gamma-ray detectors using ribbons, or "sheets" of fibers, making flexible ribbon detectors or large-area detector panels. [ 9,10] In one case, for example, over $250 \mathrm{~km}$ of $\sim 2 \mathrm{~m}$ long glass fibers were used to create a $5 \mathrm{~m}^{2}$ sensor panel.[11] Using scintillation fibers in large-area radiation instrumentation found particular traction within the community of physicists developing instruments for highenergy particle accelerator facilities.[12-14] They have also found application in health physics and homeland security applications.

In the second implementation, radiation detector developers sought to exploit the flexible, long-length scintillating fibers in the form of line detectors. In some cases small-sized detectors on the order of one $\mathrm{cm}$ in length were spliced together with standard non-scintillating fibers and used as point detectors. An application for these types of detectors is in the field of medical physics and radiation oncology, where thin diameter fibers are inserted into patients intravenously to measure the dose applied to patients during radiation therapy.[15-18] In contrast with the applications presented above, here the goal was to measure photon radiation rather than neutron radiation. In other cases long, thin fibers have been used as area photon radiation monitors for health physics protection in accelerator beam halls and at nuclear reactors.[19-23] In particular, recent research described by 
Nohtomi et al. provides a valuable starting point for considering the use of SFB detectors for nuclear security and nonproliferation applications.[23]

\section{EQUIPMENT AND METHODS}

Four SFB assemblies, each comprised of ten individual fibers, were assembled for this project using the commercial scintillating fibers BCF-10, BCF-12, and BCF-20 (Saint Gobain Crystals, Hiram, Ohio, USA).[24] The vendorsupplied technical specifications for these materials are presented in Table I. BCF-10 fibers were used to make one 2.2-m long SFB and one 14.85-m long SFB. BCF-12 and BCF-20 were used to make one SFB each; the BCF-12 SFB was $15.18-\mathrm{m}$ long and the BCF-20 SFB was $15.28-\mathrm{m}$ long. The fibers within each SFB were loosely bunched together and packaged within a light-tight, flexible vinyl tubing. The approximate outside diameters of these bundles is $0.5 \mathrm{~cm}$. The ends of the SFBs were potted within a clear plastic matrix to create a larger diameter $(1-\mathrm{cm})$ end fitting. The fibers were polished by the vendor; an example of the end of one of the SFBs is shown in Fig. 1. As a test to determine if further polishing was needed, the ends of the 2.2-m fiber were finished with a graded-approach, hand-sanding technique using 600-grit, 800-grit, and 1000-grit wet sand, and then a 1200-grit diamond wheel. After this, the ends of the SFBs were hand polished using a felt polishing wheel with ironoxide slurry. Minimal improvement was observed.

TABLE I IMPORTANT PARAMETERS FOR BCF-10, BCF-12, AND BCF-20.[24]

\begin{tabular}{|c|c|c|c|}
\hline Parameter & BCF-10 & BCF-12 & BCF-20 \\
\hline Core material & \multicolumn{3}{|c|}{ Polystyrene (common for all three) } \\
\hline Core refractive index & \multicolumn{3}{|c|}{1.60 (common for all three) } \\
\hline Density & \multicolumn{3}{|c|}{1.05 (common for all three) } \\
\hline Emission peak, nm & 432 & 435 & 492 \\
\hline Decay time, ns & 2.7 & 3.2 & 2.7 \\
\hline $1 / \mathrm{e}$ length, $\mathrm{m}^{*}$ & 2.2 & 2.7 & $>3.5$ \\
\hline$\#$ of photons per $\mathrm{MeV}^{* *}$ & $\sim 8000$ & $\sim 8000$ & $\sim 8000$ \\
\hline \multicolumn{4}{|c|}{$\begin{array}{l}* \text { For 1-mm diameter fiber; measured with a bialkali cathode } \\
\text { photomultiplier tube(PMT). } \\
* * \text { For minimum ionizing particle (MIP), corrected for PMT sensitivity. }\end{array}$} \\
\hline
\end{tabular}

Fig. 1 A magnified photo of one end of the BFC-10 SFB, showing orientation and optical polish. Note the air gap in the upper right due to epoxy wicking up into the fiber bundle, this does not affect operation. For scale note that the fibers are $1 \mathrm{~mm}$ in diameter.

The specially-made end fittings of the SFBs were designed to allow them to be easily attached or detached from a pair of Hamamatsu R9800 photomultiplier tubes (PMTs) built as Hamamatsu H10580 PMT assemblies with matching end fixtures. Technical specifications for the R9800 PMT, as provided by the vendor, are provided in Table II. A comparison of the emission spectra for the three types of fibers, and the R9800 PMT quantum efficiency spectrum are shown together in Fig. 2.[24,25]

TABLE II IMPORTANT PARAMETERS FOR THE R9800 PMT.[25]

\begin{tabular}{ll} 
Parameter & R9800 PMT \\
\hline Rise time, ns & 1.0 \\
$\begin{array}{l}\text { Transit time spread (full-width half } \\
\text { maximum, FWHM), ns }\end{array}$ & 0.270 \\
Wavelength of maximum response, nm & 420 \\
Gain & $1.1 \times 10^{6}$ \\
Photocatode material & Bialkali \\
Window material & Borosilicate glass \\
Window effective diameter, cm & 2.2 \\
\hline
\end{tabular}

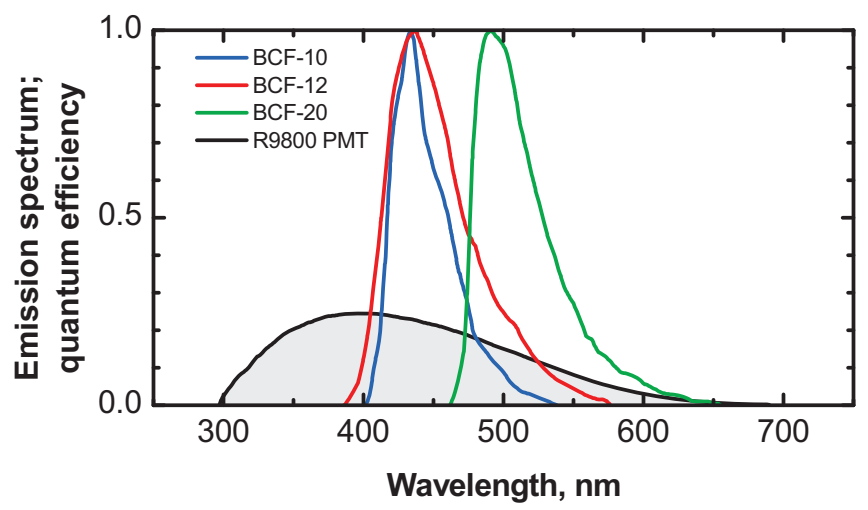

Fig. 2 The emission spectra for BCF-10, BCF-12, and BCF-20, together with the energy-dependent quantum efficiency for the R9800 PMT.[24,25]

The PMTs are housed within light-tight aluminum cases; these cases have threaded plastic fixtures in front of their photocathodes to hold and stabilize the end of a SFB. To attach the end of a SFB to the PMT module a plastic compression fitting is placed over the end of the SFB, the SFB is inserted into a hole on the PMT housing to position the SFB in front of the PMT, and the compression fitting is screwed tight onto a threaded fitting on the PMT housing. Each PMT assembly includes a transparent optical silicone pad for index matching between the SFB and PMT. A photograph of the PMT assembly, showing attachment of an SFB, is presented in Fig. 3.

Measurements were performed by digitizing the output signals of the PMT's using two channels of an Acquiris 
DC282 high-speed digitizer (4Gs/s). The DC282 was controlled by National Instruments LabView software. An application specific virtual instrument (VI) was developed using LabView that allowed the difference in arrival time of the signals between the two PMT's to be recorded, triggering off of either PMT. Slight timing errors occurred due to latency between the hardware and software derived triggers for each PMT input to the digitizer but these were $<1 \%$ of the measured signal time differences.

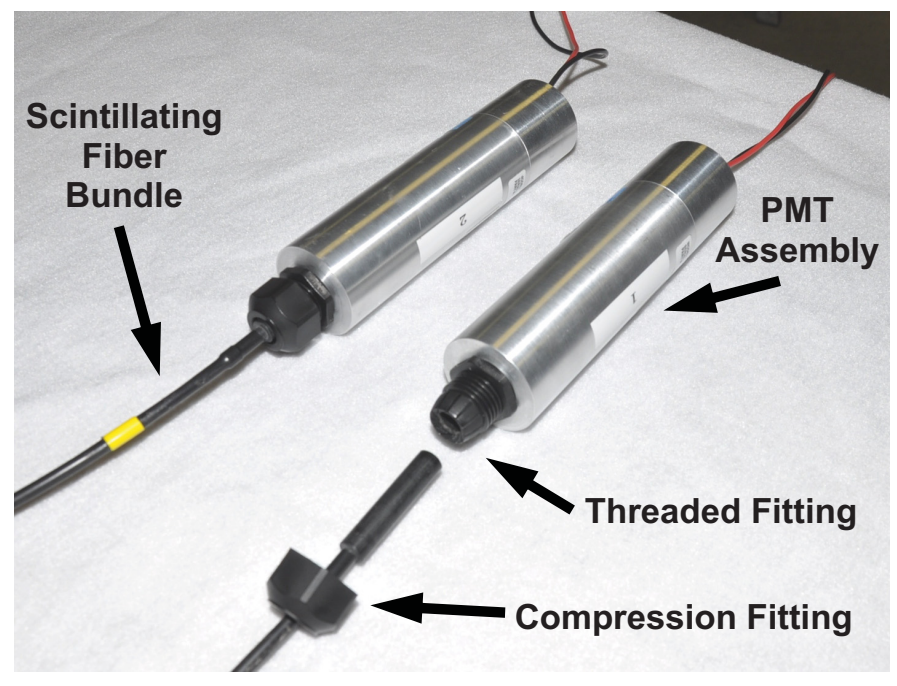

Fig. 3 A photograph of two PMT assemblies. The assembly on the left has been connected to a SFB; the assembly on the right is staged for assembly.

Prior to performing the experiments described below, the PMT's were optimized and gain matched. This was done using the 2-m BCF-10 fiber with a collimated ${ }^{60} \mathrm{Co}$ check source located in the middle of the SFB. The signal amplitudes from the two PMTs were monitored and the high voltage (HV) setting for each PMT was adjusted until both units had approximately the same output amplitude. The same procedure was implemented for the other SFBs as a system check but no further HV adjustments were necessary.

The experiments involved placing a gamma-ray check source, either an uncollimated ${ }^{60} \mathrm{Co}$ disk source $\left(3.8 \mathrm{mrem} \mathrm{hr}{ }^{-1}\right.$ (38 $\left.\mu \mathrm{Sv} \mathrm{hr}{ }^{-1}\right)$ on contact, data collected for $1800 \mathrm{~s}$ ) or a collimated ${ }^{137} \mathrm{Cs}$ disk source $\left(14 \mathrm{mrem} \mathrm{hr}^{-1}\left(140 \mu \mathrm{Sv} \mathrm{hr}{ }^{-1}\right)\right.$ on contact, data collected for $900 \mathrm{~s}$ ) adjacent to the SFBs at different locations. The position of 0 meters corresponded to the front face of one of the PMTs (PMT1). Time-difference measurements between the PMTs, made using the digitizer, were determined according to the equation $\Delta \mathrm{t}=\mathrm{t}_{\mathrm{PMT} 2}-\mathrm{t}_{\mathrm{PMT} 1}$ such that a pulse occurring closer to PMT1 (closer to position $0 \mathrm{~m}$ ) would register before the pulse reached PMT2. Because of this, pulses in the half of the SFB closer to PMT1 $(0 \mathrm{~m})$ would register with a positive time difference while those occurring in the other half of the SFB (closer to PMT2) registered as negative time differences. Pulses occurring close to the center of a SFB occurred near a time difference of zero.

\section{EXPERIMENTS ANALYZING SFB PERFORMANCE}

The primary attributes of interest when using a SFB as a linear detector are coincident pulse height amplitude (and attenuation versus path length) position linearity, position resolution, efficiency, and linearity versus rate. The following subsections present the experimental results for tests studying these characteristics.

\section{A. Coincident Pulse Height Amplitude}

A plot showing a pulse-height histogram for the Voltage signals from PMT2 using BCF-20 is presented Fig. 4. This data corresponds only to signals measured in coincidence with a signal in PMT1, data is shown for the ${ }^{60}$ Co source at $1,3,5$, $7,9,11$, and $13 \mathrm{~m}$ along the SFB. A discriminator level of 10 $\mathrm{mV}$ was used for noise rejection in the system. The most intense measurement case corresponds to having the source at the center of the SFB. With a long 1/e absorption length, BCF-20 showed the best signal-to-noise transmission towards the ends of the SFB. However, the higher scintillation light yield for BCF-10 showed a better signal-to-noise characteristic towards the center of the SFB.

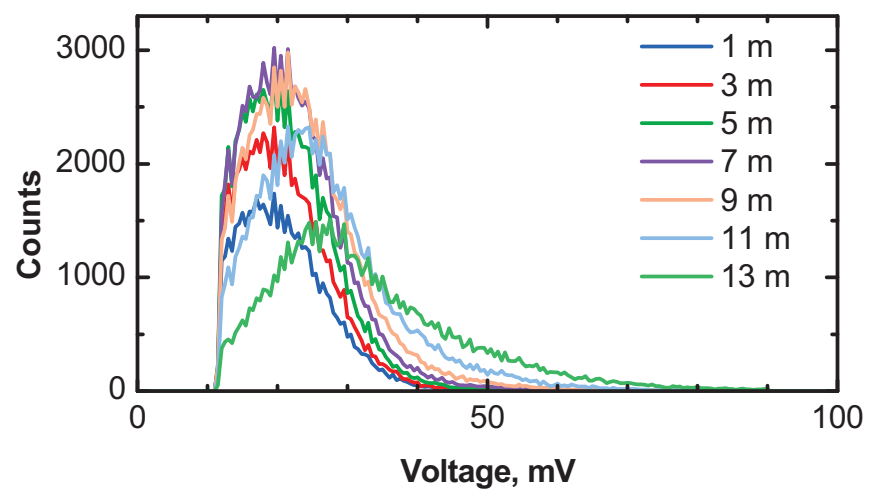

Fig. 4 Plot of the pulse-height histogram for waveforms from PMT2, when a coincident pulse is measured in the PMT1, for BCF- 20 over a $1800 \mathrm{~s}$ period using a ${ }^{60} \mathrm{Co}$ source at $1,3,5,7,9,11$, and $13 \mathrm{~m}$.

\section{B. Position Linearity}

Using the collimated ${ }^{137} \mathrm{Cs}$ source, signal time differences were recorded for $900 \mathrm{~s}$ using the three SFBs at five different locations along the length of each SFB. For BCF-10 these locations were $0.11,4.73,7.42$ (the middle), 11.14 , and 14.74 m. For BCF-12 and BCF-20, the second measurement position was $3.69 \mathrm{~m}$ instead of $4.73 \mathrm{~m}$ and the $7.42 \mathrm{~m}$ location was replaced with the middle of that fiber. Plots of the time difference for signals measured during these fifteen measurements are presented in Fig. 6. The relationship between locations along the SFB and signal time differences is clearly illustrated in these plots. The centroid position for each measurement has been plotted versus time difference in Fig. 6; the relationship is linear over the $\sim 15$-m length of these three SFBs. For this setup the relationship between position and time yields conversion relations (speed of light in the fibers) of $17.0 \pm 1.6 \mathrm{~cm} \mathrm{~ns}^{-1}$ for BCF-10, $16.8 \pm 1.6 \mathrm{~cm} \mathrm{~ns}^{-1}$ for BCF-12, and $17.0 \pm 1.6 \mathrm{~cm} \mathrm{~ns}^{-1}$ for BCF-20. This should be compared with the ideal speed of light in these plastics, determined using the index of refraction $n=1.60$ from Table I and the equation $\mathrm{v}=\mathrm{c} / \mathrm{n}$, of $\mathrm{v}=18.8 \mathrm{~cm} \mathrm{~ns}^{-1}$. The origin for the small difference between experiment and theory stems from several factors including nuances of the PMT and preamplifer responses, and the software tools used within the 
digitizer and the LabView VI to pick-off signal times. Scattering and reflection as the light transits down the length of the SFB also plays a role.[26]
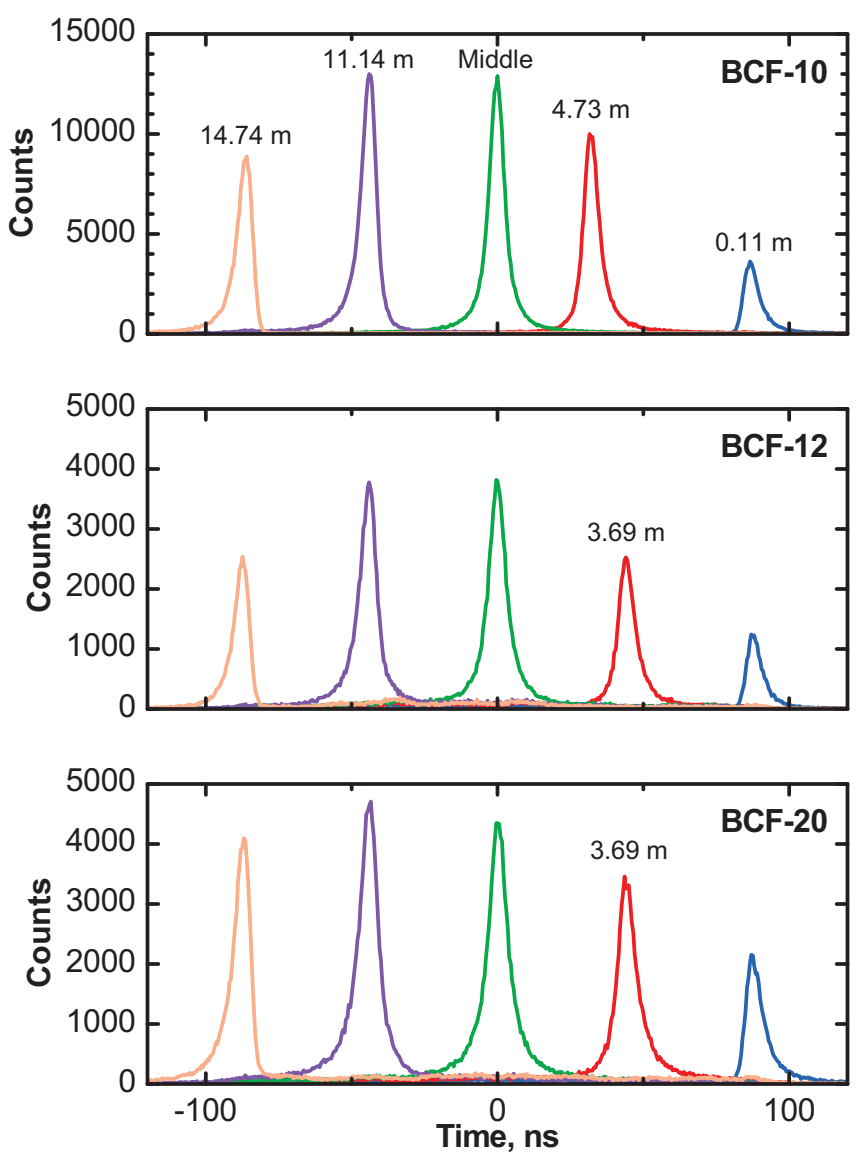

Fig. 5 These three plots show the timing resolution for measuring events in each SFB using a well-collimated ${ }^{137} \mathrm{Cs}$ source. (Note: For the BCF-12 and BCF-20 SFBs, the second measurements taken from zero (nominal $4.73 \mathrm{~m}$ for BCF-10, red trace) were taken at $3.69 \mathrm{~m}$.)

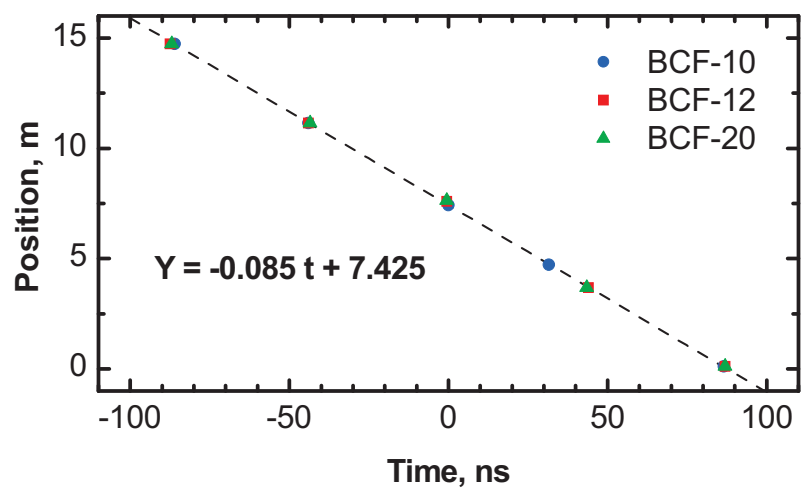

Fig. 6 Plot showing the relationship between time and position for BCF10, BCF-12, and BCF-20.

\section{Position Resolution}

The spatial resolution for these measurements is evident as the full-width half-maximum of the time differences for the individual measurements seen in Fig. 5. This information is presented in Table III. Additionally, these time difference values have been converted to distance, using the conversion factor of $8.5 \mathrm{~cm} \mathrm{~ns}^{-1}$ from Fig. 6. Overall the spatial resolution across the $15-\mathrm{m}$ long SFBs is between $50-60 \mathrm{~cm}$. The spatial resolution is slightly better towards the ends than in the middle, most likely due to the reduced effect light scattering has on the signal reaching the nearer PMT versus the farther PMT (this can be seen in the plots of Fig. 5 where the time plots near the ends of the SFBs becomes more asymmetric, with the inner edges of these distributions looking more like 'pure' Gaussian distributions.) The absolute signal intensity from the BCF-10 SBF was significantly larger than for the other two (next section.) This leads to a better signal-to-noise characteristic for these SFBs, which is likely the reason why the spatial resolution for BCF-10 is the best of the three.

TABLE III TEMPORAL AND SPATIAL Resolution OF THE BCF-10, BCF-12, AND BCF-20 SCINTILLATING FiBER BUNDLES.[24]

Resolving time (FWHM), ns ( \pm 0.5 ns)

\begin{tabular}{cccccc} 
SFB & $\mathbf{0 . 1 1} \mathbf{~ m}$ & $\mathbf{3 . 8 2} \mathbf{~ m}$ & $\mathbf{7 . 6 4} \mathbf{~ m}$ & $\mathbf{1 1 . 4 6 ~} \mathbf{~}$ & $\mathbf{1 5 . 1 7} \mathbf{~ m}$ \\
\hline BCF-10 & 6.1 & 6.2 & 6.3 & 6.2 & 6.2 \\
BCF-12 & 6.0 & 6.7 & 6.9 & 6.7 & 6.1 \\
BCF-20 & 6.2 & 7.5 & 7.8 & 7.5 & 6.6 \\
\hline \multicolumn{5}{c}{ Spatial resolution (FWHM) } & $\mathbf{c m}(\mathbf{4 . 2} \mathbf{~ c m})$ \\
SFB & $\mathbf{0 . 1 1} \mathbf{~ m}$ & $\mathbf{3 . 8 2} \mathbf{~ m}$ & $\mathbf{7 . 6 4} \mathbf{~ m}$ & $\mathbf{1 1 . 4 6 ~ \mathbf { ~ }}$ & $\mathbf{1 5 . 1 7} \mathbf{~ m}$ \\
\hline BCF-10 & 51.7 & 52.6 & 53.4 & 52.6 & 52.6 \\
BCF-12 & 50.9 & 56.8 & 58.5 & 56.8 & 51.7 \\
BCF-20 & 52.6 & 63.6 & 66.1 & 63.6 & 56.0 \\
\hline
\end{tabular}

\section{Efficiency}

The measurement efficiency for the three SFBs was determined from the fifteen measurements of Fig. 5 using the collimated ${ }^{137} \mathrm{Cs}$; this information is presented in Fig. 7. The SFBs are most sensitive in their middles, where the signal-tonoise characteristics in the PMTs are balanced. As the source is moved towards the ends it becomes more likely that, for any particular event, the signal reaching the far PMT will either be too noisy (failing the pulse-shape analysis in the LabView VI algorithm) or too weak (falling below the trigger level of the count discriminator). As presented above, the BCF-10 SFB is the most sensitive of the three tested, having a maximum efficiency of 26.9 counts s $\mathrm{s}^{-1}$ per mrem $\mathrm{hr}^{-1}$ (2.69 counts s $\mathrm{s}^{-1}$ per $\mu \mathrm{Sv} \mathrm{hr}^{-1}$.) The maximum efficiency for BCF-12 is 10.6 counts $\mathrm{s}^{-1}$ per mrem hr ${ }^{-1}$ (1.06 counts $\mathrm{s}^{-1}$ per $\mu \mathrm{Sv} \mathrm{hr}^{-1}$.) The maximum efficiency for BCF-20 is 13.9 counts $\mathrm{s}^{-1}$ per mrem $\mathrm{hr}^{-1}$ (1.39 counts s $\mathrm{s}^{-1}$ per $\mu \mathrm{Sv} \mathrm{hr}^{-1}$.) The relative efficiency of each SFB is also presented in Fig. 7. The trend of efficiency versus position along the SFB is consistent among all three sensors. It is worth noting here that the absolute efficiency likely scales with the number of fibers in the SFB, considerably more fibers could be included in a single SFB. However, this scaling may not be linear. It is possible that the total SFB efficiency may be more than the sum of the efficiency per fiber as Compton-scattered electrons generated 
in one fiber (leading to a scintillation in that fiber) have a chance to interact in nearby fibers as well. This relationship awaits further study.
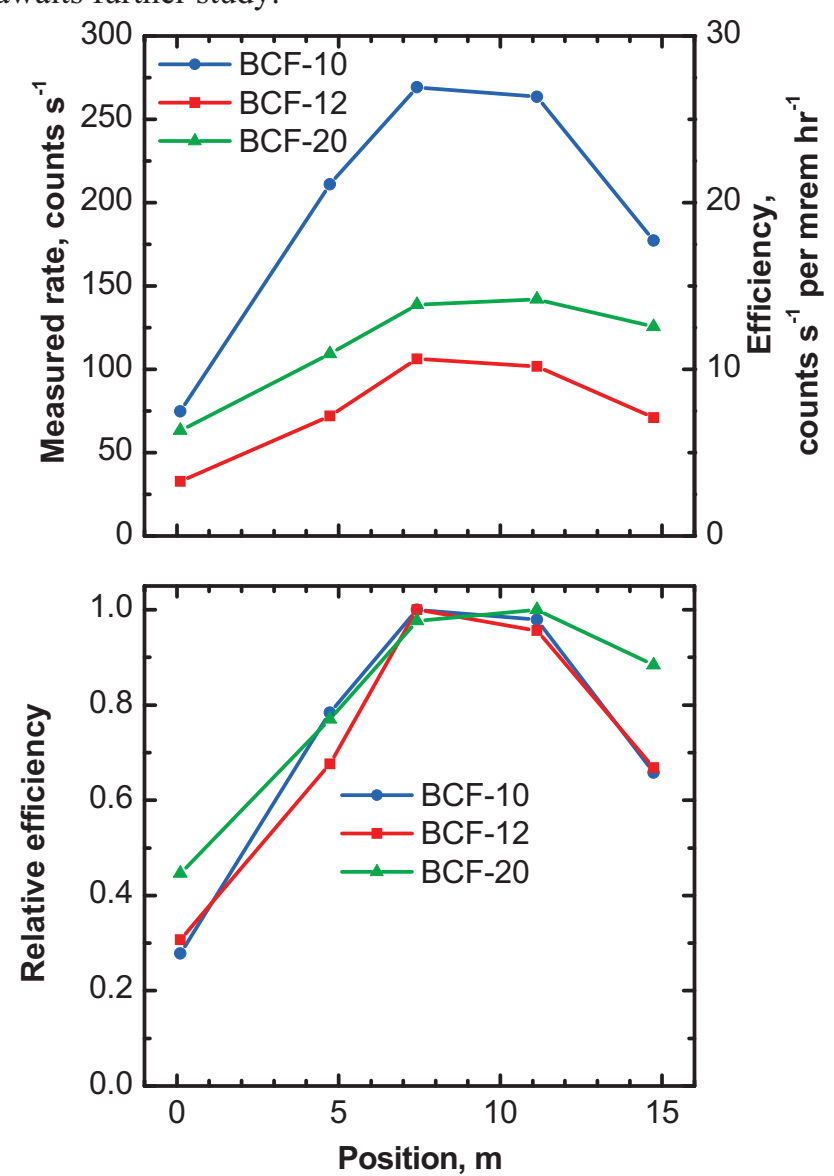

Fig. 7 The top plot shows the measured count rate, and the computed efficiency, for the three SFBs measuring a $10 \mathrm{mrem} \mathrm{hr}^{-1}{ }^{137} \mathrm{Cs}$ source. The lower plot shows the position-dependent relative efficiency, with each SFB normalized to its maximum count rate.

\section{E. Linearity Versus Rate}

A separate test was performed to examine the linearity in response for $\mathrm{BCF}-10$ versus dose rate. This test used the ${ }^{137} \mathrm{Cs}$ check source. The test started by resting the center of the SFB against the check source to achieve the maximum dose rate, $14 \mathrm{mrem} \mathrm{hr}^{-1}\left(1.4 \mathrm{mSv} \mathrm{hr}{ }^{-1}\right.$.) Tungsten plates were then placed around the check source to separate the distance between the source and SFB - decreasing the dose rate at the SFB. The maximum stand-off depth for the check source from the SFB was $6.7 \mathrm{~cm}$, producing a dose rate of $1.2 \mathrm{mrem}$ $\mathrm{hr}^{-1}\left(0.12 \mathrm{mSv} \mathrm{hr}^{-1}\right.$.) Measurements were made at seven different positions; the results are shown in Fig. 8. An acceptable $\left(\mathrm{R}^{2}=0.9965\right)$ linear fit has been applied to the $<7$ mrem $\mathrm{hr}^{-1}$ data in the plot. However, it appears that for the bare case, and the case with minimal collimation $(7 \mathrm{~mm}, 10$ mrem hr ${ }^{-1}\left(0.10 \mathrm{mSv} \mathrm{hr}^{-1}\right)$ a greater section of the SFB was exposed to the source (e.g., this collimation was not effective in exposing a uniform section of SFB). In this non-ideal case, however, the BCF-10 SFB appears to provide a conservative estimate of dose field in terms of radiological health protection. A measurement made using the ${ }^{60} \mathrm{Co}$ source is also presented on this plot.

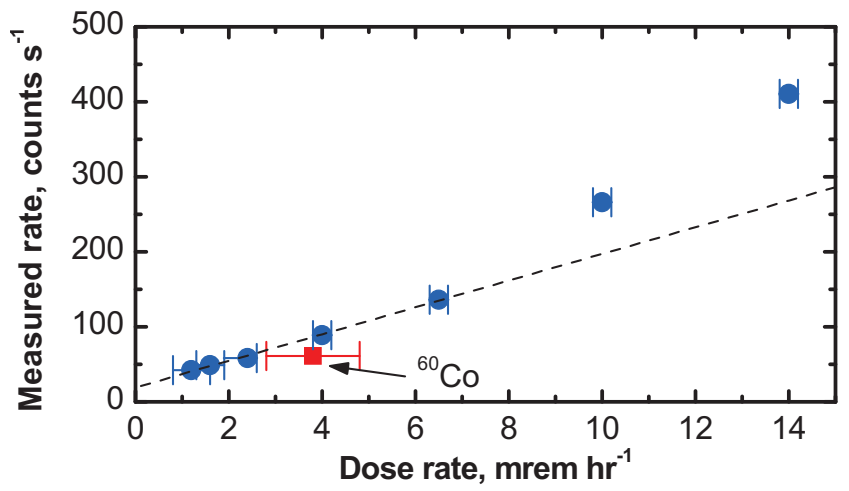

Fig. 8 Test of the linearity of the BCF-10 SFB, measured at the center of the SFB, for varying ${ }^{137} \mathrm{Cs}$ dose rates from $1.2 \mathrm{mrem} \mathrm{hr}^{-1}$ to $14 \mathrm{mrem} \mathrm{hr}^{-1}$. Also shown here is the BCF-10 SFB response to a $3.8 \mathrm{mrem} \mathrm{hr}^{-1}{ }^{60} \mathrm{Co}$ source.

\section{SUMMARY}

SFB linear detector sensors can effectively detect low-level gamma-ray radiation fields over long distances in a continuous fashion. The response is exceptionally linear over a range of $>15 \mathrm{~m}$ and they exhibit a spatial resolution of 0.5 to $0.6 \mathrm{~m}$, depending upon fiber type and position along the fiber. Their efficiency has been measured to be from 3.3 counts $\mathrm{s}^{-1}$ per mrem hr${ }^{-1}$ to 26.9 counts $\mathrm{s}^{-1}$ per mrem hr${ }^{-1}\left(0.33\right.$ counts $\mathrm{s}^{-1}$ per $\mu \mathrm{Sv} \mathrm{hr}^{-1}$ to 2.69 counts $\mathrm{s}^{-1}$ per $\mu \mathrm{Sv} \mathrm{hr}^{-1}$ ) depending upon fiber type and location along the SFB. BCF-10 is the most efficient of the three fiber-types studied, while BCF-12 is the least efficient. With the best spatial resolution as well, BCF10 appears to be the best candidate fiber, of the three studied, for use in sensor arrays of length $\sim 15 \mathrm{~m}$ or less. However, with the longest 1/e length, the BCF-20 fiber type may be a better choice for longer length sensors. Further, the performance from a BCF-20 SFB could likely be improved by choosing a different PMT with a spectral response more closely matched to the longer-wavelength output spectrum of this fiber.

Further work is needed to improve the data acquisition electronics used for this work, in particular to lower the signal noise and improve the signal-to-noise relationships and allow lowering of the lower level discrimination in the trigger logic. Further work is also justified for studying, with improved data acquisition, the limits for how long a SFB is practical for these measurements. Lastly, it should be noted that the efficiency of the SFB-type sensors used here can be easily improved by including more fibers in the SFB assemblies.

\section{ACKNOWLEDGEMENTS}

We would like to acknowledge and thank Mr. Michael Mayhugh of Saint-Gobain Crystals for his assistance preparing the SFB assemblies used in this project and for providing assistance with the concept and testing. We would also like to acknowledge and thank Prof. Akihiro Nohtomi of Kinki University, Japan, for his thoughtful suggestions and guidance early on in this project.

\section{REFERENCES}


[1] Reynolds, G. T. and Condon, P. E., "Filament Scintillation Counter," Rev. Sci. Inst. 28 (1957) 1098-1099.

[2] Chupp, E. L. and Forrest, D. J., "A Directional Neutron Detector for Space research Use," IEEE Trans. Nucl. Sci. 13 (1966) 468-477.

[3] Atkinson, M., et al., "Initial Tests of a High Resolution Scintillating Fibre (SCIFI) Tracker," Nucl. Inst. Meth. Phys. Res. A 254 (1987) 500514.

[4] Wurden, G. A., et al., "A Scintillating-Fiber 14-MeV Neutron Detector on TFTR During DT Operation," Report LA-UR-94-1604, Los Alamos National Laboratory, Los Alamos, N. M. (1994).

[5] Holslin, D., et al., "A Directional Fast Neutron Detector Using Scintillating Fibers and an Intensified CCD Camera System," Nucl. Inst. Meth. Phys. Res. A 353 (1994) 118-122.

[6] Sailor, W. C., et al., "Conceptual Design for a Scintillating-Fiber Neutron Detector for Fusion reactor Plasma Diagnostics," Rev. Sci. Inst. 66 (1995) 898-900.

[7] Ress, D., et al., "High-Sensitivity Scintillating-Fiber Imaging Detector for High-Energy Neutrons," Rev. Sci. Inst. 66 (1995) 4943-4948.

[8] Zhang, Q., Wang, Q., and Xie, Z., "Detection of Fast Neutrons Using a New Scintillating-Fiber-Array Neutron Detector," Nucl. Inst. Meth. Phys. Res. A 496 (2003) 228-232.

[9] Grazioso, R. F., et al., "Feasibility of Using Boron-Loaded Plastic Fibers for Neutron Detection," Nucl. Inst. Meth. Phys. Res. A 422 (1999) 5963.

[10] Yamashita, M., Nozaki, T., and Ninomiya, K., "Development of a Sheet Type Contamination Monitor Using the Plastic Scintillation Fiber (PSF)," Proc. 10th Congress Int. Rad. Prot. Assoc., Hiroshima, Japan, May 14-19 (2000).

[11] Abel, K. H., et al., "Scintillating-Glass-Fiber Neutron Sensors," Nucl. Inst. Meth. Phys. Res. A 353 (1994) 114-117.

[12] Ruchti, R., et al., "Development of New Scintillating Fiber Detectors for High Energy Physics Applications," IEEE Trans. Nucl. Sci 36 (1989) 146-149.

[13] Angelini, C. et al., "High-Resolution Tracking with Scintillating Fibers," Nucl. Inst. Meth. Phys. Res. A 277 (1989) 132-137.

[14] D'Ambrosio, C., et al., "Particle Tracking with Scintillating Fibers," IEEE Trans. Nucl. Sci. 43 (1996) 2115-2127.

[15] Koechner, W., "Radiation Sensitive Optical Fiber and Detector," U.S. Patent 4,788,436, November 29 (1988).

[16] Beddar, A. S., Mackie, T. R., and Attix, F. H., "Cerenkov Light Generated in Optical Fibers and Other Light Pipes Irradiated by Electron Beams," Phys. Med. Biol. 37 (1992) 925-935.

[17] Fluhs, D., et al., "Direct Reading Measurement of Absorbed Dose with Plastic Scintillators - The General Concept and Applications to Ophthalmic Plaque Dosimetry," Med. Phys. 23 (1996) 427-434.

[18] Beddar, A. S., et al., "A Miniature "Scintillator-Fiberoptic-PMT Detector System for the Dosimetry of Small Fields in Stereotactic Radiosurgery," IEEE Trans. Nucl. Sci. 48 (2001) 924-928.

[19] Imai, S.-I., et al., "New Radiation Detector of Plastic Scintillating Fiber," Rev. Sci. Inst. 62 (1991) 1093-1097.

[20] Takada, E., et al., "Neutron Radiation Distribution Sensor Using Flexible Plastic Scintillating Fiber Combined with the Time-of-Flight Technique," IEEE Trans. Nucl . Sci. 42 (1995) 570-574.

[21] Oka, T., et al., "Development of Fiber Optic Radiation Monitor Using Plastic Scintillation Fibers," J. Nucl. Sci. Tech. 35 (1998) 857-864.

[22] Makowski, D., et al., "A Distributed System for Radiation Monitoring at Linear Accelerators," IEEE Trans. Nucl. Sci. 53 (2006) 2008-2015.

[23] Nohtomi, A., et al., "On-Line Evaluation of Spatial Dose-Distribution by Using a 15m-Long Plastic Scintillation-Fiber Detector," IEEE Nucl. Sci. Symp. Conf. Rec. (2008) 965-968.

[24] "Scintillating Products, Scintillating Optical Fibers," Product Brochure, Saint Gobain Crystals, Hiram, Ohio, USA (www.detectors.saintgobain.com).

[25] "Photomultiplier Tube R9800," Product Brochure TPMH1298E04, Hamamatsu Photonics, Iwata City, Shizuoka Prefecture, Japan (2010).

[26] Zugec, P., "A Timing Resolution Model for Scintillating Fibers," IEEE Nucl. Sci. Symp. Conf. Rec. (2011) 1605-1608. 